\section{An overview of risk factors associated to post-partum depression in Asia}

\author{
Shubham Mehta, ${ }^{1}$ Nidhi Mehta² \\ 1Department of Psychiatry, SMS Medical \\ College, Jaipur; 2Department of \\ Obstetrics and Gynaecology, Bangalore \\ Medical College and Research Institute, \\ Bengaluru, India
}

\begin{abstract}
Post partum depression (PPD) is an important complication of child-bearing. It requires urgent interventions as it can have long-term adverse consequences if ignored, for both mother and child. If PPD has to be prevented by a public health intervention, the recognition and timely identification of its risk factors is must. We in this review have tried to synthesize the results of Asian studies examining the risk factors of PPD. Some risk factors, which are unique to Asian culture, have also been identified and discussed. We emphasize on early identification of these risk factors as most of these are modifiable and this can have significant implications in prevention of emergence of post partum depression, a serious health issue of Asian women.
\end{abstract}

\section{Introduction}

Post-partum depression (PPD) is the most common complication of childbearing, occurring in $10-15 \%$ of women after delivery. ${ }^{1}$ It usually begins within the first six weeks post partum and represents a considerable public health problem affecting women and their families. ${ }^{2}$

The effects of postnatal depression on the mother, her married life, and her children make it an important condition to diagnose, treat and prevent. ${ }^{3}$ Whilst very severe postnatal depression can easily be diagnosed, less severe presentations of depressive illness can be easily dismissed as normal or natural phenomena of childbirth. Consequently, this untreated postpartum depression can have long-term adverse effects, both on mother and her children. If PPD is to be prevented by clinical or public health intervention, its risk factors (i.e. factors which could significantly predict the occurrence of PPD) need to be faithfully identified. Studies in the developing world have found that risk factors are often culturally determined. This review aims at improving our understanding of the culturally determined risk factors of PPD within Asian culture.

\section{Materials and Methods}

We conducted a literature search using PubMed and PsychINFO databases. The keywords used were risk factors, post partum, postnatal, after child birth, Asia and depression. Articles published in English language or providing an abstract (with complete information) in English were included in this study. The studies included have to be the original research articles in the form of observational cohorts and surveys. All the articles which focused on factors associated to PPD in Asian context were then selected. The factors which showed significant association with development of PPD were considered as risk factors for PPD. Assessment of PPD could be done both by structured clinical interview and selfrated questionnaire in studies selected. The studies that relied on special subgroups (e.g., pregnant or postpartum women with HIV infection) were eliminated; 27 studies were thus identified and the data regarding the number of subjects, mode and time period of assessment were further extracted from the identified studies, which are mentioned further.

\section{Results and Discussion}

Number of risk factors were identified on analyzing the selected studies. The following table (Table 1) ${ }^{4-30}$ shows the list of studies which have analyzed the possible risk factors for PPD in Asian context. The risk factors thus identified from various studies can be grouped as following categories.

\section{Demographic factors}

Among these, the factors which have shown significant association with PPD are age of mother at the time of child birth as well as older age at marriage. ${ }^{4}$ Second, being migrant and giving birth to child overseas has also been identified as a risk factor for PPD. In a study examining Japanese women, who were born and raised in Japan but who gave birth to their child in Hawaii, USA, half of the participants experienced emotional dysfunction during their pregnancy. All primipara females experienced post-partum depression. The participants who had maternity blues tended to have PPD. 5 Another study assessing the incidence of PPD symptomatology in a sample of immigrant Asian Indian women found that there was a minor depressive symptomatology rate of $28 \%$ and an additional major depressive symptomatology rate of $24 \%{ }^{6}$ Different health care attitudes in different cultures and distance from family leading to homesickness could be the possible reasons. However, no consistent association of PPD has been found
Correspondence: Shubham Mehta, Department of Psychiatry, SMS Medical College, Room No. 9, N.M.H.P. Trainees Hostel, Psychiatric Centre Campus, Sethi Colony, Jaipur 302004, Rajasthan, India.

Tel. +91.979.972.3735

E-mail: drshubhammehta@gmail.com

Key words: post partum, post natal depression, after child birth, risk factors, Asia.

Contributions: the authors contributed equally.

Conflict of interests: the authors declare no potential conflict of interests.

Received for publication: 25 February 2014.

Revision received: 3 April 2014.

Accepted for publication: 4 April 2014.

This work is licensed under a Creative Commons Attribution NonCommercial 3.0 License (CC BYNC 3.0).

(c) Copyright S. Mehta and N. Mehta, 2014

Licensee PAGEPress, Italy

Mental Illness 2014; 6:5370

doi:10.4081/mi.2014.5370

with maternal education. ${ }^{7}$ Lower socio-economic status has been found to be another factor associated with PPD. Although pregnancy and childbirth are generally viewed as a time of joy and pleasure in most of the families, they also put on financial burden in form of expenses for a new member of the family, especially, among low-income families or nuclear families, where the husband is the only one who provides family income. ${ }^{8,9}$ This can cause depression in new mothers. Only one study has found the association of religion with PPD. 10

\section{Clinical factors}

An important factor which can lead to PPD is parity. It has been observed that frequency of primi-parity is higher in women with PPD. 5,11 Having 5 or more children was responsible for persistence of prenatal depression beyond the first few postnatal months. ${ }^{8}$ On the contrary, one study found significant association of multi-parity with PPD. 12

Another significant factor is unplanned/ unwanted or negative attitude toward pregnancy. 13,14 A study by Limlomwongse and Liabsuetrakul (2006) found that negative attitudes towards this pregnancy double the risk of PPD. ${ }^{10}$ Premarital pregnancy is another risk factor for PPD which is important in context of Asian countries. It is considered highly unacceptable in most Asian culture, reason being a highly conservative attitude toward sex among Asian people than people in the west. Getting pregnant before marriage may reflect that the woman had experienced a premarital sexual intercourse, which is considered as a shame or 
taboo in many of the Asian countries. ${ }^{31-35}$ However, unplanned or unwanted pregnancy as a risk factor for PPD should be interpreted very cautiously. It merely reflects the circumstances in which the pregnancy occurred and not the feeling of woman towards the growing fetus.

History of premenstrual symptoms, ${ }^{15,16}$ previous depression or having depression during pregnancy, ${ }^{9,12}$ prenatal high anxiety, ${ }^{14}$ history of post-partum, 17 or maternity blues, $, 5,16$ have all been consistently demonstrated as putative risk factors for PPD. The studies examining these factors provide preliminary evidence that some hormone-related phenomena are related to the occurrence of post-partum mood disorders. The results in a way support the notion that the etiology for post-partum mood disorders may be related to differential hormonal sensitivity. Such risk factors should be carefully assessed and evaluated clinically in detail when dealing with a woman of PPD.

Others include physical ill-health, ${ }^{7}$ pregnancy complications or woman's perception of having complications during this pregnancy, 10 preterm delivery, ${ }^{18}$ and history of pregnancy loss. 12

A study by Dindar and Erdogan (2007) found smoking to be significant risk factor for PPD in Turkish women. ${ }^{9}$

However, there are some contradictory views in literature too. In one study, risk of PPD was not found to be related to age, level of education, employment status, planned/ unplanned pregnancy, history of abortion and pregnancy-related complications, term and type of delivery, gender of the child, and mother's breast-feeding and other reporting no relation between method of delivery and risk of PPD. ${ }^{7,11}$

\section{Psychosocial factors}

Psychosocial factors that were found to be associated with postnatal depression are living in mixed/conflicting influences of culture, 19 poor accommodation, 20 lack of social support, ${ }^{17,21,22}$ lack of instrumental support or medical resources, ${ }^{23}$ stressful life events during pregnancy,9,15 lack of confidant/friend, 7 conflicts/being abused by in-laws, $, 12,24$ and conflicts with relatives over child care. ${ }^{23}$

There are different types of social support, for example informational support (where advice and guidance is given), instrumental

Table 1. Risk factors associated to post-partum depression.

\begin{tabular}{|c|c|c|c|c|}
\hline Author & Country & $\begin{array}{c}\text { Overall } \\
\text { sample size }\end{array}$ & $\begin{array}{l}\text { Mode of } \\
\text { assessment }\end{array}$ & $\begin{array}{l}\text { Time of } \\
\text { assessment }\end{array}$ \\
\hline Green et al. ${ }^{4}$ & United Arab Emirates & 125 & EPDS & 3 and 6 months postpartum \\
\hline Taniguchi et al. ${ }^{5}$ & Japanese migrants in USA & 45 & Telephonic interview & Within 1 year of child-birth \\
\hline Goyal et al. ${ }^{6}$ & Asian-Indian immigrants in Australia & 58 & PPD screening scale & $\begin{array}{l}\text { Between } 2 \text { weeks and } 12 \\
\text { months postpartum }\end{array}$ \\
\hline Small et al. 7 & Vietnamese, Turkish and Filipino immigrant & 318 & Personal interviews & N/A \\
\hline Rahman et al. ${ }^{8}$ & Pakistan & 160 & SRQ, BDQ, modified Life Events Checklist & $\begin{array}{l}\text { At } 3,6 \text { and } 12 \text { months } \\
\text { postpartum }\end{array}$ \\
\hline Dindar et al. ${ }^{9}$ & Turkey & 679 & EPDS, Risk Factor questionnaire & Within first year after delivery \\
\hline Limlomwongse et al. ${ }^{10}$ & Thailand & 610 & EPDS & 6-8 weeks postpartum \\
\hline Akman et al. 11 & Turkey & 302 & Structured clinical interview & $\begin{array}{l}\text { First day of child birth and } 6 \\
\text { weeks postpartum }\end{array}$ \\
\hline Ho-Yen et al. 12 & Norway & 426 & EPDS & 5-10 weeks after delivery \\
\hline Andajani-Sutjahjo et al. ${ }^{13}$ & Indonesia & 41 & EPDS & 6 weeks postpartum \\
\hline Sayil et al. 14 & Turkey & 200 & Structured interview & 6-8 months postpartum \\
\hline Aydin et al. 15 & Turkey & 728 & EPDS, Structured interview & Within 1st postnatal year \\
\hline Bloch et al. ${ }^{16}$ & Israel & 1800 & Structured interview & 6-8 weeks postpartum \\
\hline Ayvaz et al. 17 & Turkey & 316 & EPDS, GHI, BAI, BDI & $\begin{array}{l}6-8 \text { weeks and } 6 \text { months } \\
\text { postpartum }\end{array}$ \\
\hline Tamaki et al. ${ }^{18}$ & Japan & 627 & EPDS, STAIS & 1,3 and 4 months after delivery \\
\hline Leung et al. ${ }^{19}$ & Hong-Kong and Chinese & 11 & Structured interview & 6 months postpartum \\
\hline Kitamura et al. ${ }^{20}$ & Japan & 290 & DSM-III-R criteria for depression & Within 3 months of delivery \\
\hline Liabsuetrakul et al.21 & Thailand & 400 & PDRS, DSM-IV for depression & 6-8 weeks postpartum \\
\hline Husain et al. ${ }^{22}$ & Pakistan & 149 & EPDS, MSPSS, PIQ & 12 weeks postpartum \\
\hline Chee et al. ${ }^{23}$ & Singapore & 559 & $\begin{array}{l}\text { Screening questionnaire, } \\
\text { structured interview }\end{array}$ & 6 weeks postpartum \\
\hline Lee et al. ${ }^{24}$ & Chinese women in Hong-Kong & 959 & EPDS & $\begin{array}{l}\text { Immediately after delivery, } \\
3 \text { months postpartum }\end{array}$ \\
\hline Danaci et al. ${ }^{25}$ & Turkey & 317 & EPDS & 6 months postpartum \\
\hline Fisher et al. ${ }^{26}$ & Vietnam & 506 & Structured interview, EPDS & 6 weeks postpartum \\
\hline Rodrigues et al. .27 & India & 39 & EPDS & N/A \\
\hline Azidah et al. ${ }^{28}$ & Malaysia & 421 & $\begin{array}{l}\text { EPDS, questionnaire on psychosocial } \\
\text { support and traditional post natal care }\end{array}$ & $\begin{array}{l}1 \text { week and } 4-6 \text { weeks } \\
\text { postpartum }\end{array}$ \\
\hline Leung et al. ${ }^{29}$ & Hong-Kong Chinese women & 385 & EPDS & N/A \\
\hline Sabuncuoglu et al. 30 & Turkey & 80 & EPDS, AAQ & $\begin{array}{l}\text { Within } 2 \text { to } 18 \text { months } \\
\text { postpartum }\end{array}$ \\
\hline
\end{tabular}

PPD, post-partum depression; EPDS, Edinburgh postnatal depression scale; BAI, Beck's anxiety inventory; BDI, Beck's depression inventory; GHI, general health inventory; STAIS, State trait anxiety inventory state; PDRS, postpartum depression risk scale; MSPSS, multidimensional scale of perceived social support; PIQ, personal information questionnaire; DSM, diagnostic and statistical manual of mental disorders 
support (practical help in terms of material aid or assistance with tasks) and emotional support (expressions of caring and esteem). As social support has been demonstrated to be important in transition to motherhood and has an impact on emotional coping, lack of such social support can be a potent predictor for postpartum depression in some women. ${ }^{21,23}$

Conflicts between mother- and daughter-inlaw are notoriously common in Asian societies. $^{24}$ In Asian societies, traditionally, marriage means a daughter-in-law joining the family and adjusting accordingly rather than composing a new household for the newlyweds. The daughter-in-law is commonly entrusted to the supervision and control of her mother-in-law, who is generally portrayed as authoritarian. ${ }^{24}$ Thus, appropriately some studies have demonstrated mother-in-law conflicts as a significant problem among married women in those countries. ${ }^{25,36}$ These conflicts may stand responsible for emergence of PPD.

\section{Husband/marriage related factors}

The factors which fall into this category are psychiatric illness in husband, 25 current alcoholism, ${ }^{9}$ poor educational status, ${ }^{8}$ uncertainty about husband's work/unemployment, 13,15 husband's polygamous relationships (9), disturbed relationships with husband or marital conflict, ${ }^{12,14}$ lack of support from husband, ${ }^{15,26}$ regret for marriage, 13 and low involvement of husband over child care. ${ }^{27}$

\section{Child-related factors}

Regarding child-related factors, health problems of child, 15 dissatisfaction with child's gender (birth of a girl child),12,21 birth defects in child, 13 child's temper tantrums, 15 child's feeding difficulties, ${ }^{7}$ stress with child care, ${ }^{28,29}$ and only short period of rest/exhaustion after childbirth, ${ }^{26}$ were all associated with PPD. In terms of gender of the child, there are quite a few studies that suggest dissatisfaction in infant's gender (birth of a baby girl) is amongst the risk factors for postpartum depression. This implies the significance of infant's gender in Asian Family. In some Asian cultures, married couples are expected by their family to have at least one son to maintain the continuity of the bloodline. ${ }^{36}$ In Turkey, a baby boy is seen as a source of income. Women who cannot give birth to a baby boy may be considered incapable, leading to serious problems in the marriage. 12

Logically, variables relating to the child can be measured postpartum only. It has been found that mothers suffering from postpartum depression give more negative descriptions of their children than control mothers and report more behavioral problems in their children. ${ }^{37}$ Therefore, the mothers' symptoms may be a source of bias in the reporting of child characteristics and the results of such studies examining child-related factors must be viewed with caution.

\section{Miscellaneous}

Poor self body image with weight conscience, ${ }^{4}$ personality disorders (e.g. avoidant, dependent, and obsessive-compulsive), ${ }^{11}$ insecure attachment style, ${ }^{20}$ are the other factors which have been shown to increase the risk of emergence of PPD in women.

\section{Conclusions}

There are several risk factors for this highly prevalent problem of postpartum depression in Asian countries, some of which are unique to Asian culture. It is likely that a interplay of these factors play a role in the causation of postpartum depression. Taking care of these largely modifiable risk factors can prevent development of postpartum depression. A collaborative-care approach (for example, collaboration between a mental-health professional and an obstetrician) would be reasonable to identify mothers who are at high-risk for development of PPD. Resolution of marital and family conflicts before conception, helping the mothers to establish a support plan, have realistic expectations of birth and parenting, addressing self-esteem issues and encouraging them to quit smoking could be some of the ways to prevent PPD.

Although an over-simplification, following scenario can portray the results from the synthesis of literature on risk factors of postpartum depression and can help in better understanding of the results: her clinical history may reveal previous experience of psychiatric illness, and she may have suffered from depressive or anxious symptoms during pregnancy. She may be experiencing difficulties through stressful life events and a poor marital relationship. She perceives that her partner, family and friends are not as supportive as they could be.

\section{References}

1. 0'Hara MW, Swain AM. Rates and risk of postpartum depression-a meta-analysis. Int Rev Psychiatr 1996;8:37-54.

2. Setse R, Grogan R, Pham L, et al. Longitudinal study of depressive symptoms and healthrelated quality of life during pregnancy and after delivery. Maternal Child Health J 2009;13:577-87.

3. Robinson GE, Stewart DE. Postpartum disorders. In: Stotland NL, Stewart DE, eds. Psychological aspects of women's health care. 2nd ed. Washington, DC: American Psychiatric Press, Inc.; 2001. pp 117-139.

4. Green K, Broome H, Mirabella J. Postnatal depression among mothers in the United Arab Emirates: sociocultural and physical factors. Psychol Health Med 2006;11:42531.

5. Taniguchi H, Baruffi G. Childbirth overseas: the experience of Japanese women in Hawaii. Nurs Health Sci 2007;9:90-5.

6. Goyal D, Murphy SO, Cohen J. Immigrant Asian Indian women and postpartum depression. J Obstet Gynecol Neonatal Nurs 2006;35:98-104.

7. Small R, Lumley J, Yelland J. Cross-cultural experiences of maternal depression: associations and contributing factors for Vietnamese, Turkish and Filipino immigrant women in Victoria, Australia. Ethn Health 2003;8:189-206.

8. Rahman A, Creed F. Outcome of prenatal depression and risk factors associated with persistence in the first postnatal year: prospective study from Rawalpindi, Pakistan. J Affect Disord 2007;100:115-21.

9. Dindar I, Erdogan S. Screening of Turkish women for postpartum depression within the first postpartum year: the risk profile of a community sample. Public Health Nurs 2007;24:176-83.

10. Limlomwongse N, Liabsuetrakul T. Cohort study of depressive moods in Thai women during late pregnancy and 6-8 weeks of postpartum using the Edinburgh postnatal depression scale (EPDS). Arch Womens Ment Health 2006;9:131-8.

11. Akman C, Uguz F, Kaya N. Postpartumonset major depression is associated with personality disorders. Compr Psychiatry 2007:48:343-7.

12. Ho-Yen SD, Bondevik GT, Eberhard-Gran M, Bjorvatn B. Factors associated with depressive symptoms among postnatal women in Nepal. Acta Obstet Gynecol Scand 2007;86:291-7.

13. Andajani-Sutjahjo S, Manderson L, Astbury J. Complex emotions, complex problems: understanding the experiences of perinatal depression among new mothers in urban Indonesia. Cult Med Psychiatry 2007;31:101-22.

14. Sayil M, Gure A, Ucanok Z. First time mothers' anxiety and depressive symptoms across the transition to motherhood: associations with maternal and environmental characteristics. Women Health 2006;44:61-77.

15. Aydin N, Inandi T, Karabulut N. Depression and associated factors among women within their first postnatal year in Erzurum province in eastern Turkey. Women Health 2005;41:1-12.

16. Bloch M, Rotenberg N, Koren D, Klein E. Risk factors associated with the development of postpartum mood disorders. J Affect Disord 2005;88:9-18.

17. Ayvaz S, Hocaoglu C, Tiryaki A, Ak I. Incidence of postpartum depression in Trabzon province and risk factors at gesta- 
tion. Turk Psikiyatri Derg 2006;17:243-51.

18. Tamaki R, Murata M, Okano T. Risk factors for postpartum depression in Japan. Psychiatry Clin Neurosci 1997;51:93-8.

19. Leung S, Arthur DG, Martinson I. Stress in women with postpartum depression: a phenomenological study. J Adv Nurs 2005; 51:353-60.

20. Kitamura T, Yoshida K, Okano T et al. Multicentre prospective study of perinatal depression in Japan: incidence and correlates of antenatal and postnatal depression. Arch Womens Ment Health 2006;9:121-30.

21. Liabsuetrakul T, Vittayanont A, Pitanupong J. Clinical applications of anxiety, social support, stressors, and self-esteem measured during pregnancy and postpartum for screening postpartum depression in Thai women. J Obstet Gynaecol Res 2007; $33: 333-40$.

22. Husain N, Bevc I, Husain M, et al. Prevalence and social correlates of postnatal depression in a low income country. Arch Womens Ment Health 2006;9:197202.

23. Chee CY, Lee DT, Chong YS, et al. Confinement and other psychosocial factors in perinatal depression: a transcultural study in Singapore. J Affect Disord 2005;89:157-66.

24. Lee DT, Yip AS, Leung TY, Chung TK. Ethnoepidemiology of postnatal depression. Prospective multivariate study of sociocultural risk factors in a Chinese population in Hong Kong. Br $\mathrm{J}$ Psychiatry 2004;184:34-40.

25. Danaci AE, Dinc G, Deveci A, et al. Postnatal depression in turkey: epidemiological and cultural aspects. Soc Psychiatry Psychiatr Epidemiol 2002;37:125-9.

26. Fisher JR, Morrow MM, Ngoc NT, Anh LT. Prevalence, nature, severity and correlates of postpartum depressive symptoms in Vietnam. BJOG 2004;111:1353-60.

27. Rodrigues M, Patel V, Jaswal S, et al. Listening to mothers: qualitative studies on motherhood and depression from Goa, India. Soc Sci Med 2003;57:1797-806.

28. Azidah AK, Shaiful BI, Rusli N, Jamil MY. Postnatal depression and socio-cultural practices among postnatal mothers in Kota Bahru, Kelantan, Malaysia. Med J Malaysia 2006;61:76-83.

29. Leung SS, Martinson IM, Arthur D. Postpartum depression and related psychosocial variables in Hong Kong Chinese women: findings from a prospective study. Res Nurs Health 2005;28:27-38.

30. Sabuncuoglu 0, Berkem M. Relationship between attachment style and depressive symptoms in postpartum women: findings from Turkey. Turk Psikiyatri Derg 2006;17:252-8.

31. Xia DY, Liao SS, He QY, et al. A questionnaire-based survey on attitude and behavior of sex among rural women in Hainan province. Zhonghua Liu Xing Bing Xue Za
Zhi 2004:25:586-9.

32. Alexander M, Garda L, Kanade S, et al. Correlates of premarital relationships among unmarried youth in Pune district, Maharashtra, India. Int Fam Plan Perspect 2007;33:150-9.

33. Hojat M, Shapurian R, Nayerahmadi H, et al. Premarital sexual, child rearing, and family attitudes of Iranian men and women in the United States and in Iran. J Psychol 1999;133:19-31.

34. Somrongthong R, Panuwatsuk P, Amarathithada D, et al. Sexual behaviors and opinions on sexuality of adolescents in a slum community in Bangkok. Southeast Asian J Trop Med Public Health 2003;34:443-6.

35. Cha ES, Doswell WM, Kim KH, et al. Evaluating the theory of planned behavior to explain intention to engage in premarital sex amongst Korean college students: a questionnaire survey. Int J Nurs Stud 2007; 44: 1147-1157.

36. Kim J, Buist A. Postnatal depression: a Korean perspective. Australas Psychiatry 2005;13:68-71.

37. Murray, L. Effects of postnatal depression on infant development: direct studies of early motherinfant interactions. In: Kumar $\mathrm{R}$, Brockington IF, eds. Motherhood and mental ilness 2: causes and consequences. London: Wright; 1988. 